Cite this: Nanoscale, 2013, 5, 5262

Received 14th March 2013 Accepted 10th April 2013

DOI: $10.1039 / c 3 n r 01289 a$

\section{A microfluidic origami chip for synthesis of functionalized polymeric nanoparticles $\uparrow$}

\author{
Jiashu Sun,,$^{\mathrm{a}}$ Yunlei Xianyu, ${ }^{\mathrm{a}}$ Mengmeng Li,,$^{\mathrm{a}}$ Wenwen Liu, ${ }^{\mathrm{a}}$ Lu Zhang, ${ }^{\mathrm{a}}$ \\ Dingbin Liu, ${ }^{a}$ Chao Liu, ${ }^{b}$ Guoqing $\mathrm{Hu}^{\mathrm{b}}$ and Xingyu Jiang ${ }^{* a}$
}

www.rsc.org/nanoscale

This report demonstrates a microfluidic origami chip to synthesize monodisperse, doxorubicin-loaded poly(lactic-co-glycolic acid) nanoparticles with diameters of $\sim 100 \mathrm{~nm}$, a size optimized for cellular uptake and anticancer efficacy, but difficult to achieve with existing approaches. This three-dimensional design in a microchannel may allow for the fabrication of polymeric nanoparticles in this size regime with ease.

Functionalized polymeric nanoparticles hold great promise as potential drug delivery vehicles in terms of controlled drug release, reduced cell toxicity, and better therapeutic efficacy. ${ }^{\mathbf{1 , 2}}$ Among a variety of polymeric materials that can be used for preparing nanoparticles, poly(lactic-co-glycolic acid) (PLGA) has attracted particular attention due to its optimal properties such as biocompatibility and biodegradability. ${ }^{3}$ PLGA nanoparticles have been successfully employed to deliver drugs and bioactive agents in vivo. ${ }^{4}$ The loaded drug can be released from PLGA nanoparticles in a controlled and sustained manner upon degradation of the polymer. ${ }^{5}$ A critical parameter in evaluating the performance of PLGA nanoparticles is the particle size. ${ }^{6}$ Nanoparticles smaller than $100 \mathrm{~nm}$ have a prolonged circulation half-time than those larger than $200 \mathrm{~nm}$. The smaller nanoparticles also show a higher rate of cellular uptake than larger ones. ${ }^{7,8}$ Conventional approaches for preparing drugloaded PLGA nanoparticles include emulsion-solvent evaporation, nanoprecipitation and polymerization. ${ }^{9}$ Among these techniques, nanoprecipitation can easily produce small PLGA nanoparticles $(<100 \mathrm{~nm})$ by dissolving the polymer and drug in organic solution followed by adding aqueous solution. However, these PLGA nanoparticles have a broad size

${ }^{a}$ CAS Key Lab for Biological Effects of Nanomaterials and Nanosafety, National Center for NanoScience and Technology, Beijing, 100190, China. E-mail: xingyujiang@ nanoctr.cn

${ }^{b}$ LNM, Institute of Mechanics, Chinese Academy of Sciences, 100190, China

$\dagger$ Electronic supplementary information (ESI) available: See DOI: 10.1039/c3nr01289a

‡ These authors contributed equally to this work. distribution as a result of poor control over mixing in bulk. Microfluidics-based methods allow rapid mixing and precise control of different streams, and provide a simple means to prepare polymeric nanoparticles with narrow size distribution. ${ }^{\mathbf{1 0 - 1 2}}$ For example, hydrodynamic flow focusing and passive mixing enhanced by particular microstructures have been applied to fabricate homogeneous PLGA-PEG nanoparticles with diameters of tens of nanometers. ${ }^{13}$ In this work we propose a microfluidic origami chip with different geometries that enables rapid mixing of PLGA-doxorubicin (DOX) solution and water, resulting in monodisperse DOX-loaded PLGA nanoparticles with a controllable size from 70 to $230 \mathrm{~nm}$ in a single nanoprecipitation step. The cellular uptake and anticancer efficiency of synthesized PLGA-DOX nanoparticles are investigated. This foldable microfluidic origami chip is highly versatile and can be applied to prepare a variety of polymeric nanoparticles by nanoprecipitation.

The microfluidic origami chip is made by bonding two polydimethylsiloxane (PDMS) layers after oxygen plasma. The two $500 \mu \mathrm{m}$ thick PDMS layers are obtained by spin-coating uncured PDMS on the surface of silicon wafer at $500 \mathrm{rpm}$ for $30 \mathrm{~s}$. The top layer contains an embedded microfluidic channel $(300 \times 300 \mu \mathrm{m}$ cross-section and $6 \mathrm{~cm}$ long $)$ with three inlets and one outlet. The bottom layer is a flat PDMS slab. The assembled origami chip can be manually folded to form different geometries such as arc and double spiral (Fig. 1 and $\mathrm{S} 1$, for details see $\mathrm{ESI} \dagger) .{ }^{14-16}$

In the experiments, PLGA-DOX nanoparticles were precipitated through rapid mixing of one organic stream in the middle inlet channel and two water streams in the side inlet channels (Fig. 1). The organic solution (2\% PLGA-DOX) was prepared by dissolving PLGA and DOX in the mixture of dimethylformamide (DMF) and trifluoroethanol (TFE). The 2\% PLGA-DOX solution was introduced into the microchannels from the middle inlet at varying flow rates from $0.3125 \mathrm{~mL} \mathrm{~h}^{-1}$ to $2.5 \mathrm{~mL} \mathrm{~h}^{-1}$, and hydrodynamically focused into a thin stream by two rapidly flowing water sheaths $\left(20 \mathrm{~mL} \mathrm{~h}^{-1}\right.$ for each water stream). The ratio of the flow rate of organic solution to the total flow rate 
(a)

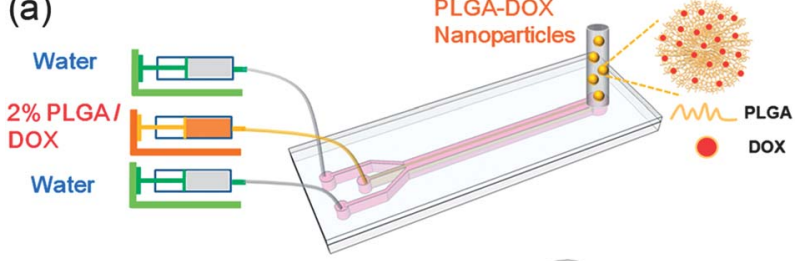

(b)

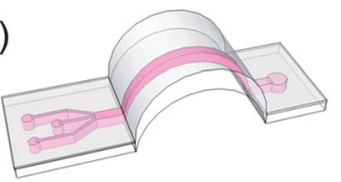

(c)

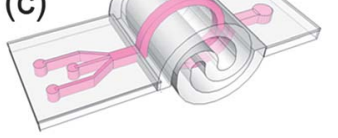

Fig. 1 (a) Schematics of a microfluidic origami chip for synthesizing monodisperse DOX-loaded PLGA nanoparticles. (b) An arc geometry obtained by manually folding the origami chip. (c) A double spiral geometry obtained from the same chip.

(the flow ratio, $R$ ) was $7.75 \times 10^{-3}-0.059$ by adjusting the flow rate of $2 \%$ PLGA-DOX solution (Table S1 $\dagger$ ).

The origami chip in two-dimensional (2D) flat geometry was first applied to synthesize PLGA-DOX nanoparticles of different sizes by varying the flow rate of organic solution (Fig. 1(a)). At a low flow ratio $(R \leq 0.059)$ and small Reynolds number $(\mathrm{Re} \approx$ 60 ), the focused narrow organic stream would rapidly mix with water through diffusion. The mixing time by diffusion $\left(\tau_{\text {mix,diff }}\right)$ was $0.59 \mathrm{~ms}$ at the flow ratio of $7.75 \times 10^{-3}$, and prolonged to $30.86 \mathrm{~ms}$ with the increased flow ratio of $0.059 .^{7}$ The mixing time $\left(\tau_{\text {mix }}\right)$ was also evaluated with a three-dimensional (3D) numerical simulation using Fluent, which was in good agreement with $\tau_{\text {mix,diff }}$ (Table S2, see ESI $\dagger$ ).

The precipitation of PLGA-DOX nanoparticles occurred as soon as the organic stream diffused into water streams. ${ }^{17}$ The time of formation of PLGA-DOX nanoparticles was estimated to be around 10-54 ms, ensuring an almost complete solvent displacement of DMF and TFE (see ESI $\dagger$ ). ${ }^{13}$ The size of PLGADOX nanoparticles synthesized by the flat microchannel at different flow ratios was measured using dynamic light scattering, and confirmed by scanning electron microscopy (SEM) (Fig. 2(b) and (c)). We observed that the particle size increased from $100 \pm 1.18 \mathrm{~nm}$ to $234 \pm 3.38 \mathrm{~nm}$ in an approximately exponential trend with the increasing flow rate of the organic stream (Fig. S2 $\dagger$ ). We also noted that the polydispersity index (PDI) in all measurements was less than 0.13, indicating a monodisperse dispersion of PLGA-DOX nanoparticles (Fig. S3†).

To further decrease the mixing time and synthesize smaller PLGA-DOX nanoparticles, we manually folded the origami chip to form a 3D geometry such as an arc or a double spiral (Fig. S1†). The mixing distance and mixing time inside 3D geometries were predicted by 3D models using Fluent. The simulation results indicated that the combination of hydrodynamic focusing and 3D curved microchannels could significantly shorten the mixing distance and reduce the mixing time (Fig. 3(a) and (b) and S4 $\uparrow$ ). For instance, at the flow rate of $2.5 \mathrm{~mL} \mathrm{~h}^{-1}(R=0.059)$ for organic solution, the mixing time was $16 \mathrm{~ms}$ in 3D arc microchannels and $14.5 \mathrm{~ms}$ in 3D double spiral microchannels, approximately $46 \%$ faster than that in 2D flat
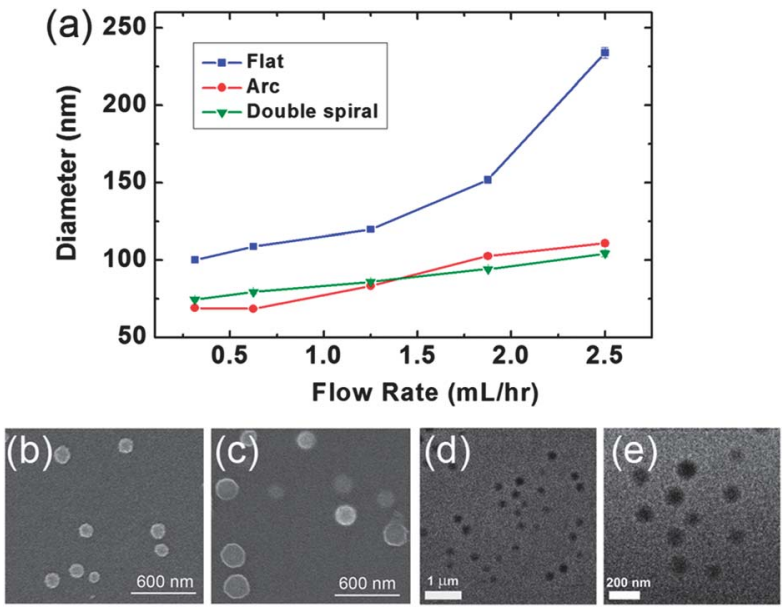

Fig. 2 (a) Size distribution of PLGA-DOX nanoparticles as a function of flow rate of the organic stream in the origami chip with different geometries. (b and c) SEM images of nanoparticles synthesized in 2D flat channels at $0.375 \mathrm{~mL} \mathrm{~h}^{-1}$ and $2.5 \mathrm{~mL} \mathrm{~h}^{-1}$, respectively. ( $\mathrm{d}$ and e) TEM images of nanoparticles synthesized in 3D double spiral channels at $2.5 \mathrm{~mL} \mathrm{~h}^{-1}$.

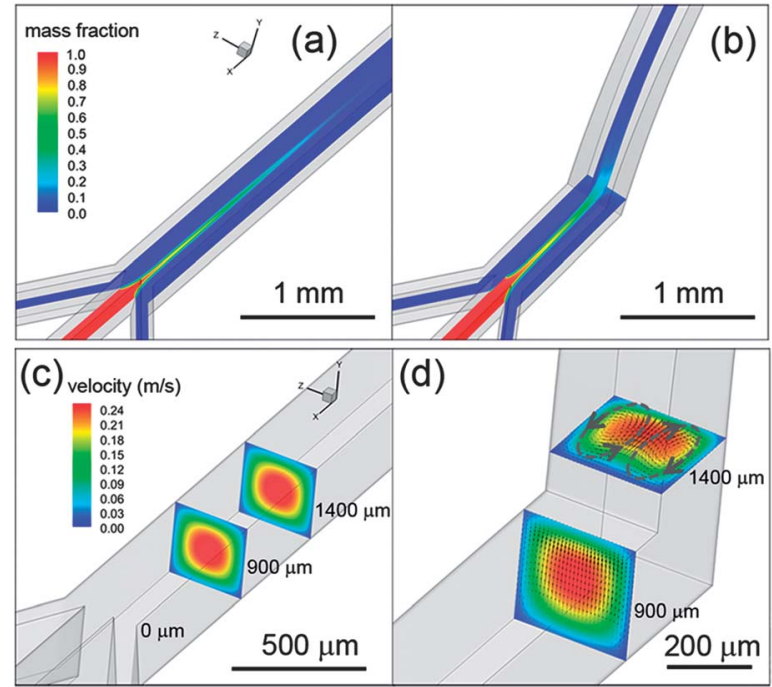

Fig. 3 Simulating prediction of rapid mixing in (a) 2D flat microchannels, and (b) $3 \mathrm{D}$ double spiral microchannels at $2.5 \mathrm{~mL} \mathrm{~h}^{-1}$. Comparison of the velocity magnitude at the same position inside (c) 2D flat microchannels, and (d) 3D double spiral channels at $2.5 \mathrm{~mL} \mathrm{~h}^{-1}$ for organic solution. The small black arrows in (d) represent the velocity vector projected onto the cross-section, indicating two counter-rotating vortices at $1400 \mu \mathrm{m}$ which are highlighted by two dasheddotted ellipses in dark gray.

microchannels (Table S2 $\dagger$ ). This was due to 3D origami structures resulting in two counter-rotating vortices perpendicular to the flow direction that could efficiently increase the mixing after curved turns (Fig. 3(d)). ${ }^{10}$ This enhanced mixing resulted in the generation of small PLGA-DOX nanoparticles $(\leq 100 \mathrm{~nm})$ with a monodisperse distribution (PDI $<0.06$ ) at a high flow rate of $2.5 \mathrm{~mL} \mathrm{~h}^{-1}(R=0.059)$ inside 3D double spiral microchannels. The prepared nanoparticles were observed by transmission electron microscopy (TEM) (Fig. 2(d) and (e)). In comparison, to prepare $100 \mathrm{~nm}$ nanoparticles inside 2D flat microchannels, we 
need to use a low flow rate of $0.3125 \mathrm{~mL} \mathrm{~h}^{-1}$ for organic solution (8 times lower than that in 3D channels), while the PDI of synthesized nanoparticles was 0.13 (two times larger than that in $3 \mathrm{D}$ channels). Such improvement in 3D channels suggested a better throughput of the nanoparticle synthesis. Moreover, the manually folded 3D channels had smooth turns to avoid abrupt velocity changes, ensuring that no clogging occurs inside microchannels for over several hours. ${ }^{18}$

The cellular uptake of PLGA-DOX nanoparticles was compared against free DOX solution by using two human carcinoma cell lines, MCF-7 and HeLa. The PLGA-DOX nanoparticles of $100 \mathrm{~nm}$ in size were synthesized by 3D double spiral microchannels at $2.5 \mathrm{~mL} \mathrm{~h}^{-1}$. The DOX concentration in nanoparticles was around $50 \mu \mathrm{g} \mathrm{mL}^{-1}$ which was determined by measuring the absorption of PLGA-DOX nanoparticle solution at $480 \mathrm{~nm}$ (Fig. S5 $\dagger$ ). The encapsulation efficiency was around $50 \%$ by using the microfluidic origami chip. To achieve a final DOX concentration of $10 \mu \mathrm{g} \mathrm{mL} \mathrm{m}^{-1}$ inside nanoparticles, the suspension was diluted by using high-glucose Dulbecco's modified Eagle's medium (DMEM). After co-incubation of free DOX or PLGA-DOX nanoparticles at a DOX concentration of $10 \mu \mathrm{g} \mathrm{mL}{ }^{-1}$ with MCF-7 or HeLa cells for $4 \mathrm{~h}$ at $37^{\circ} \mathrm{C}$, cells were washed twice with phosphate buffered saline (PBS) before fluorescence observation. ${ }^{19}$ Fig. 4 (a) shows confocal fluorescent microscopic images of MCF-7 and HeLa cells after $4 \mathrm{~h}$ treatment. Both MCF-7 and HeLa cells incubated with PLGA-DOX nanoparticles displayed higher green fluorescence intensities than those treated with free DOX, indicating an enhanced cellular uptake of PLGA-DOX nanoparticles. This might be because nanoparticles were taken up by means of an endocytic process, while free DOX permeated the cell membrane through passive diffusion. ${ }^{5}$

To investigate the effect of PLGA-DOX nanoparticles on the cancer cell cytotoxicity, MCF-7 and HeLa cells were co-incubated with $100 \mathrm{~nm}$ nanoparticles of different DOX concentrations for a set time period at $37{ }^{\circ} \mathrm{C}$. Cancer cells were also treated with free DOX at equal concentrations to compare the therapeutic efficacy between the drug-loaded nanoparticles and the free drug. The viability of MCF-7 and HeLa cells incubated with PLGA-DOX nanoparticles and free DOX was measured using cell counting kit-8 (CCK-8). The changes in cell morphology as a function of incubation time were recorded with a microscope. For two different cell lines, most cancer cells treated with PLGADOX nanoparticles exhibited higher cytotoxicity than that of free DOX (Fig. 4(b) and (c)). The viability of MCF-7 cells was higher than $90 \%$ after incubation with free DOX at $2-20 \mu \mathrm{g}$ $\mathrm{mL}^{-1}$ for $12 \mathrm{~h}$. In comparison, the cell viability decreased to $64.52 \pm 15.11 \%$ (at a DOX concentration of $20 \mu \mathrm{g} \mathrm{mL}^{-1}$ ) in a dose-dependent manner after MCF-7 cells were co-incubated with PLGA-DOX nanoparticles for $12 \mathrm{~h}$. Moreover, drug-loaded nanoparticles could dramatically decrease the cell viability by 58.2-100\% for MCF-7 cells, and 73.8-97.9\% for HeLa cells after $24 \mathrm{~h}$ incubation. Microscopic images of cancer cells at different incubation times revealed that nanoparticles, even at low DOX concentrations $\left(\leq 5 \mu \mathrm{g} \mathrm{mL} \mathrm{mL}^{-1}\right.$ ) would alter the cell morphology and cause cell death, consistent with the viability test (Fig. S6 and $\mathrm{S} 7 \dagger$ ). We should note that PLGA-DOX nanoparticles showed a rapid release profile of DOX in an incubation time of 3 (a)

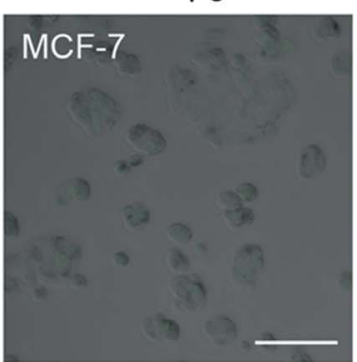

(b)

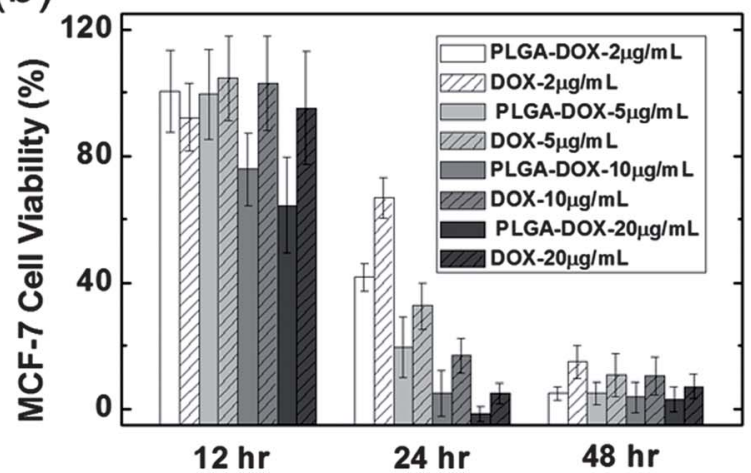

DOX-10 $\mu \mathrm{g} / \mathrm{mL}$

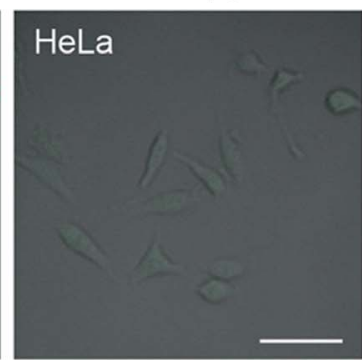

(c)

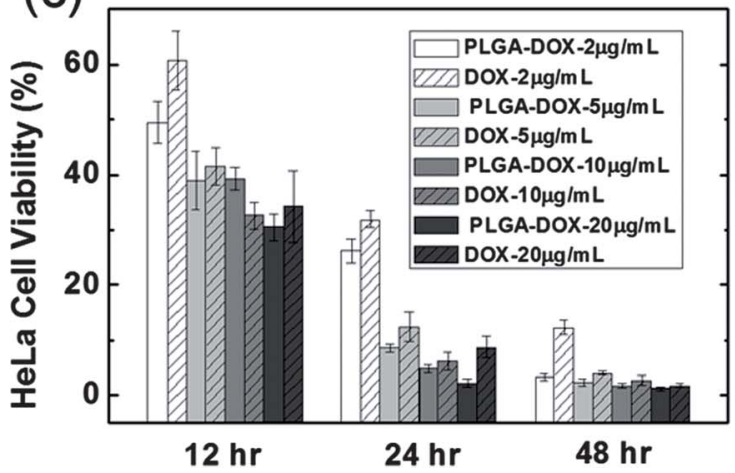

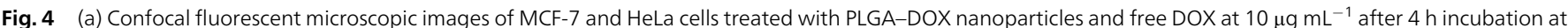

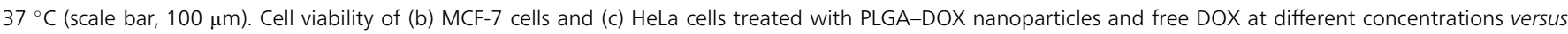
incubation time. Standard deviations are obtained from six replicas. 
days. ${ }^{20}$ A previous study showed that the monodisperse PLGA nanoparticles of $100 \mathrm{~nm}$ had an optimal performance of cellular uptake. ${ }^{21}$ In our case, the internalized drug-loaded PLGA nanoparticles by endocytosis could provide a high local concentration of DOX, thus increasing the cell cytotoxicity. ${ }^{22}$ In addition, free DOX diffused into cancer cells might be pumped out by $p$-glycoprotein due to the multi-drug resistance (MDR) effect, ${ }^{19,23}$ while PLGA-DOX nanoparticles could be immune from this effect. ${ }^{5}$

Our microfluidic origami chip with various 3D geometries has been applied to synthesize monodisperse functionalized nanoparticles through enhanced mixing in 3D microchannels. The prepared PLGA-DOX nanoparticles were around $100 \mathrm{~nm}$ with a monodisperse distribution, exhibiting an improved cellular uptake and anticancer efficacy. The throughput of nanoparticle synthesis varied with the flow rate of the organic solution. At a maximum flow rate of $2.5 \mathrm{~mL} \mathrm{~h}^{-1}$ for $2 \%$ PLGADOX solution, the origami chip produced $1200 \mathrm{mg}$ of nanoparticles per day. To increase the synthesis throughput, designing of parallel microfluidic channels should be attempted in the future study. This versatile microfluidic chip would be extended for preparing other polymeric nanoparticles by a single-step nanoprecipitation.

J. S. and X. J. acknowledge financial support from MOST (2009CB930001, 2011CB933201 and 2012AA030308), NSFC (51105086, 21025520, 21025520 and 51073045), CAS (KJCX2YW-M15), and SKLEAC201207. G. H. acknowledges financial support from MOST (2011CB707604), NSFC (11272321), and CAS (KJCX2-YW-H18).

\section{Notes and references}

1 M. E. Davis, Z. Chen and D. M. Shin, Nat. Rev. Drug Discovery, 2008, 7, 771-782.

2 D. Liu, W. Chen, K. Sun, K. Deng, W. Zhang, Z. Wang and X. Jiang, Angew. Chem., Int. Ed., 2011, 50, 4103-4107.

3 R. A. Jain, Biomaterials, 2000, 21, 2475-2490.

4 K. A. Woodrow, Y. Cu, C. J. Booth, J. K. Saucier-Sawyer, M. J. Wood and W. M. Saltzman, Nat. Mater., 2009, 8, 526533.
5 H. S. Yoo and T. G. Park, J. Controlled Release, 2001, 70, 63-70.

6 F. Alexis, E. Pridgen, L. K. Molnar and O. C. Farokhzad, Mol. Pharmaceutics, 2008, 5, 505-515.

7 R. Karnik, F. Gu, P. Basto, C. Cannizzaro, L. Dean, W. KyeiManu, R. Langer and O. C. Farokhzad, Nano Lett., 2008, 8, 2906-2912.

8 D. Liu, Z. Wang and X. Jiang, Nanoscale, 2011, 3, 1421-1433.

9 M. L. Hans and A. M. Lowman, Curr. Opin. Solid State Mater. Sci., 2002, 6, 319-327.

10 J. S. Sun, C. Liu, M. M. Li, J. D. Wang, Y. L. Xianyu, G. Q. Hu and X. Y. Jiang, Biomicrofluidics, 2013, 7, 011802.

11 C. H. Fang, L. Shao, Y. H. Zhao, J. F. Wang and H. K. Wu, Adv. Mater., 2012, 24, 94-98.

12 A. Sauret and H. C. Shum, Appl. Phys. Lett., 2012, 100, 154106.

13 P. M. Valencia, P. A. Basto, L. F. Zhang, M. Rhee, R. Langer, O. C. Farokhzad and R. Karnik, ACS Nano, 2010, 4, 16711679.

14 B. Yuan, Y. Jin, Y. Sun, D. Wang, J. S. Sun, Z. Wang, W. Zhang and X. Y. Jiang, Adv. Mater., 2012, 24, 890-896.

15 J. Sun, Y. Kang, E. Boczko and X. Jiang, Electroanalysis, 2013, 25, 1023-1028.

16 J. Sun, M. Li, C. Liu, Y. Zhang, D. Liu, W. Liu, G. Hu and X. Jiang, Lab Chip, 2012, 12, 3952-3960.

17 U. Bilati, E. Allemann and E. Doelker, Eur. J. Pharm. Sci., 2005, 24, 67-75.

18 M. Rhee, P. M. Valencia, M. I. Rodriguez, R. Langer, O. C. Farokhzad and R. Karnik, Adv. Mater., 2011, 23, H79$\mathrm{H} 83$.

19 X. J. Li, V. Ling and P. C. H. Li, Anal. Chem., 2008, 80, 40954102.

20 H. S. Yoo, J. E. Oh, K. H. Lee and T. G. Park, Pharm. Res., 1999, 16, 1114-1118.

21 A. R. Xu, M. F. Yao, G. K. Xu, J. Y. Ying, W. C. Ma, B. Li and Y. Jin, Int. J. Nanomed., 2012, 7, 3547-3554.

22 T. H. Tran, C. T. Nguyen, D. P. Kim, Y. K. Lee and K. M. Huh, Lab Chip, 2012, 12, 589-594.

23 X. J. Li, Y. C. Chen and P. C. H. Li, Lab Chip, 2011, 11, 13781384. 